International Journal of Engineering \& Technology, $7(2.33)(2018) 657-659$
International Journal of Engineering \& Technology
WPC
Website: www.sciencepubco.com/index.php/IJET
Research paper

\title{
An inventive arrangement for accident prevention detection and caution using image mining
}

\author{
Stephen raj. $\mathrm{S}^{1 *}$, Sripriya. $\mathbf{P}^{2}$ \\ ${ }^{1}$ Ph.D scholar, Department of Computer Applications, Vels University, Chennai, Tamil nadu, India. \\ ${ }^{2}$ Associate Professor, Department of Computer Applications, Vels University, Chennai, Tamil nadu, India \\ *Corresponding author E-mail: chanraj9@gmail.com
}

\begin{abstract}
Driver aware is more main cause for the majority accidents connected to motor vehicle crashes. Lethargic, tried, heavy-eyed driver identification methods can form the base of a classification to potentially decrease accidents linked to driver tiredness. It obtain visual cue such as eyelid progress, look movement, skull movement, and facial look that naturally distinguish the intensity of awareness of a human being are extract in actual time and analytically united to assume the exhaustion intensity of the driver. A probabilistic model is used for predict human being in-alertness based on the image cues obtained. The real-time use of several image cues and their regular arrangement yields a much more healthy and exact exhaustion and distress characterization than by a single image cue. Percent eye conclusion is also unwavering. It is deemed to be logically robust, dependable and faithful in exhaustion and fright categorization, finding and caution.
\end{abstract}

Keywords: Computer Vision; Tiredness Detection; Face Detection; Alerts.

\section{Introduction}

Nationalized public road travel Safety management scrutiny data indicate that drowsiness whilst dynamic is a causal issue intended for highway accident and it outcome within 4-6 time top crash risk family member to attentive drivers .the majority of the deadly road accidents happen at speeds improved than $70 \mathrm{Kmph}$. The World Health Organization has reported that India has the most awful road situation in the world resulting roughly two and a half million death in 2010 and 2016 [3] [5].

Study show that driver exhaustion and sleepiness is single of the main reason for the growing accidents. Driver exhaustion not merely impact the watchfulness and reply point to the starter but it enhances probability of organism concerned in vehicle accident. These drowsy drivers are unsuccessful to get right procedures prior to a crash. A significant satire in driver's exhaustion is to the driver may be too exhausted to understand his own intensity of sleepiness. This important difficulty is often unseen by the drivers. Accordingly, the use of behind system that checks a driver's level of watchfulness is essential to evade highway accidents. These systems must aware the driver in the case of drowsiness or carelessness. Some caution code that can be

deliberate as indication of driver exhaustion are inattention while on the highway, driving larger than the centre row, sensation impatient, feeling firm, heavy eye and reacting gradually. There has been concentrated research to detect sleepiness of drivers, based on the above mention gesture of cadaver (i.e. eye action detection). Here an wellorganized driver's tiredness recognition system is intended using eye blinking detection by taking eye detection into consideration so that highway accident can be avoid effectively [2] [4].

\section{Conditions study}

[14] It contain composed navigation performance statistics and process them toward lock away exhaustion impaired pattern by with image actions for characteristic mining. They contain conduct the research through a heavy simulation. The vehicle producers Saab have planned a new creation.

[15] Mouthful air taster of starter facing the start motor four wheels. Afterward the AlcoKey's radio teller passing a warning sign to the vehicle electronic manage component to let happening base on the liquor height in the mouthful of air taster. Those researches employ the connections among soul and motor means of transportation to point toward intoxicated lashing. Their instrument require to change the motor four wheel and be firmly joined among the supplementary add on, so there is compromise.

[16] Heitmann et al. contain intended sequence a diversity of skill, such as skull place feeler, eyeball scheme, two trainees based systems and in chair trembling scheme, for watchfulness monitor and endorsement. This technology, they enclose worn watch and check driver exhaustion. However, their system is very complex in the new phase, for instance the eyeball system and trainee base scheme is motionless firm to exist incorporated into the motor four wheelers.

\section{Study of driver's health condition}

The research has recognized cue characteristic energetic performance used for intoxicated driver. These efforts, our review this drunken and tiredness starting connected obey into 3 category. These primary and next categories centers on starting obeys connected to motor four wheels progress it, such group draw the faction tendency the 3 rd process is concerning the starter health condition 
linked toward slanted decision plus notice of the starter. We near these 3 category health condition as below [1] [19].

i) Cue connected to path location preservation problems: such as weaving, traveling, swerve, and rotating suddenly, unlawfully or with a broad radius [7] [19].

ii) Cue connected to momentum direct problems: such as accelerate or decelerate abruptly, braking randomly and stop unsuitably [7] [14].

iii) Cue connected to ruling and caution problems: such as heavy with tiredness on middle or path indicator, heavy on the other side of the highway, next toward intimately, heavy without headlights at nighttime, and slow respond to traffic signal [7] [8] [12].

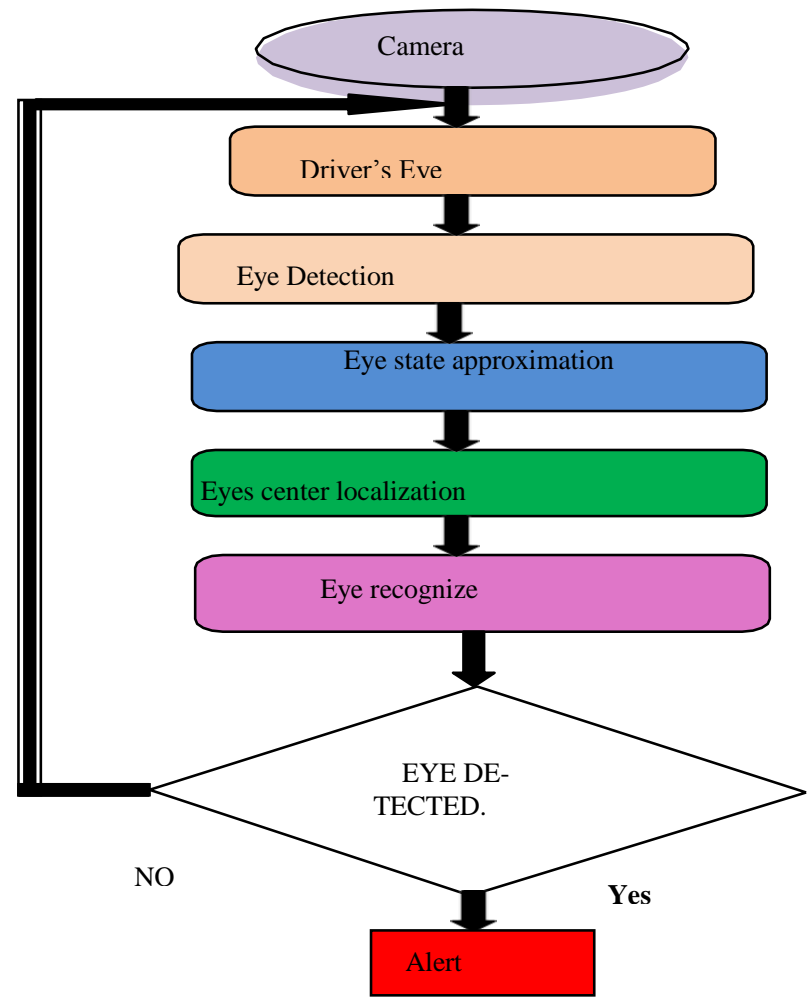

Fig. 1: Block Diagram.

\section{Explanation of research}

Eye region are silent too big for credit). We were reducing its area by crop only area approximately the eye middle. To restrict the eyeball middle, we adopt the technique for localize the eye middle and suggest round object discovery by judgment cosine approach between unit slope vector coldness vector that can be intended by each aspirant[6][9].

If aspirant position is situated at the middle of surrounding, the chilliness vector will have same way. It resources cosine between two vectors nearly 1 and lesser value for additional points[10].

$\mathrm{J}_{\mathrm{i}}=\sum \cos \theta / \mathrm{M}$

The site of eyeball center is location that has utmost value Sc. After we get site of eyeball middle, we will harvest area approximately eye center for gratitude [11] [13].

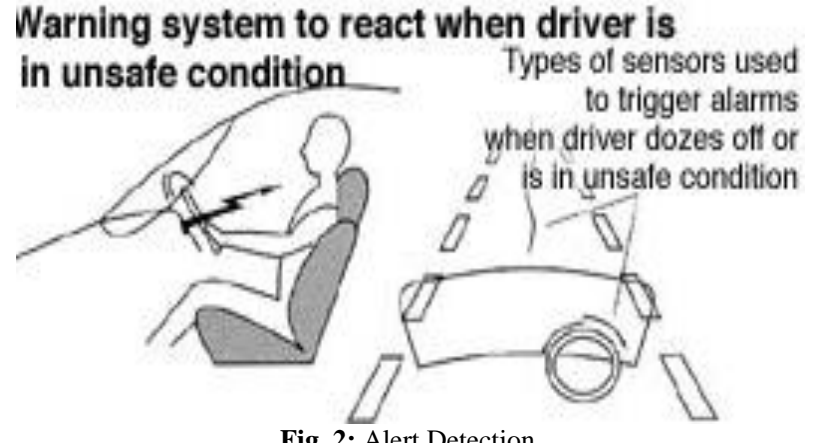

\section{Eyeball localization}

If the driver meet with a mishap previous to wake up then the accident is also detect in this research by allowing for the prevention its check if driver able to drive or not. It is cautious as an accident and a message is send to the predefined get in touch with person. A contact list is set with the contact numbers of friends or relatives to ensure them that the person is not in a condition to drive more so that they can make remedies for that. . Escape dread of the pc can be used as the fear for the demand. A chronometer is set next the driver is detecting as sleepiness, which is an enormously little value for manufacture the fear as soon as possible. The anxiety, ceaselessly create echo till the driver wake up and put it off in the flesh [15] [17]

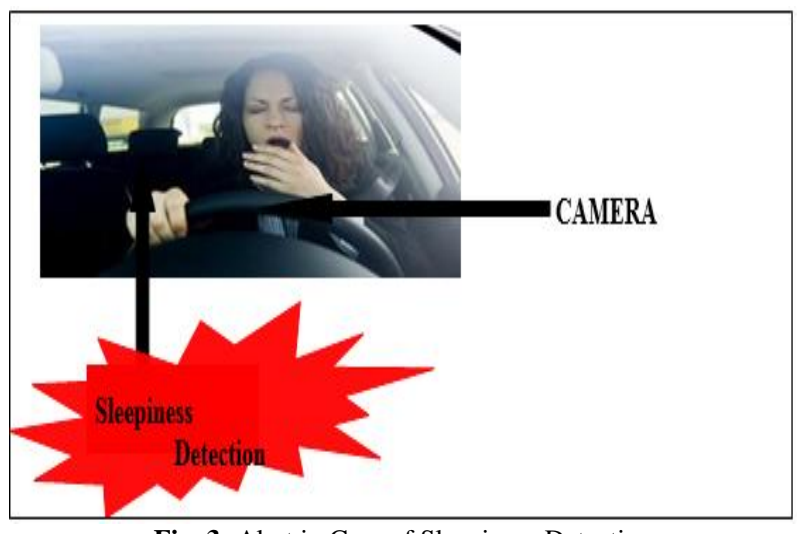

Fig. 3: Alert in Case of Sleepiness Detection

\section{Conclusion}

In this manuscript, we suggest a sleepiness detection technique using the cross connection between space and slope vectors. We primary detect a human being face based on the Heer features. The eyeball region can be roughly resolute within the detected eye area. Inside the eyeball region, we place a dark circular object correspond to a beginner. Since the coldness and incline vectors are normalize in the calculation of cross connection, the technique works well despite of the intensities of an contribution image. This help to detect the beginner beneath different lighting circumstances. The computational cost of the planned method is low and it works at image rate. As prospect work, we prepare to examine the proposed method on more image sequences in more diversify lighting circumstances.

\section{References}

[1] A. Amditis, M. Bimpas, G. Thomaidis, M. Tsogas, M. Netto, S.Mammar, A. Beutner, N. Möhler, T.Wirthgen, S. Zipser, A. Etemad,M. Da Lio, and R. Cicilloni, "A Situation-Adaptive Lane Keeping Support System: Overview of the SAFELANE approach", in Proc. IEEE Trans. Intell. Transp. Syst., vol. 11, no. 3, September 2010.

[2] Jaeik Jo, Ho Gi Jung, Kang Ryoung, Jaihie Kim, "Vision -based method for detecting driver drowsiness and distraction in driver 
monitoring system", in Proc. Optical Engineering, vol 15, no.12, December 2012.

[3] Kohji Murata, Etsunori Fujita, Shigeyuki Kojima, Shinitirou Maeda Yumi Ogura, Tsutomu Kamei, Toshio Tsuji, "Noninvasive Biological Sensor System for Detection of Drunk Driving", in Proc. IEEE Trans. Info. Tech. Biomedicine, vol. 15, no. 1, January 2011.

[4] Paul Viola Michael J. Jones Daniel Snow,'Detecting Pedestrians Using Patterns of Motion and Appearance", in Proc. of the Ninth IEEE International Conference on Computer Vision (ICCV'03) 0-76951950-4/03.

[5] Zibo Li, Guangmin Sun, Fan Zhang, Linan Jia, Kun Zheng, Dequn Zhao", "Smartphone Based Fatigue Detection System using Progressive Locating Method", in Proc.IET Intell. Transp. Syst., pp. 1-9, January 2015.

[6] Bappaditya Mandal, Liyuan Li, Gang Sam Wang, and Jie Lin, "Towards Detection of Bus Driver Fatigue Based on Robust Visual Analysis of Eye State", in Proc. IEEE Trans. Intell. Transp. Syst., vol. 18, no. 3, March 2013.

[7] Behnoosh Hariri, Shabnam Abtahi, Shervin Shirmohammadi, Luc Martel., "Demo:Vision Based Smart in-Car Camera System for Driver Yawning Detection", in Proc. IEEE Trans. Intell. Transp. Syst., 2016.

[8] P. M. Forsman, B. J. Vila, R. A. Short, C. G. Mott, and H. P. A. van Dongen," Efficient Driver Drowsiness Detection at Moderate Levels of Drowsiness", International Journal of Accident Analysis and Prevention, page no:341- 350, September 2012.

[9] Vandna Saini, Rekha Saini, "Driver Drowsiness Detection System and Techniques: A Review “, in Proc of. IJCSIT Vol. 5 (3), 2014, 4245-4249.

[10] Z. Zhu and Q. Ji, "Real Time and Non-intrusive Driver Fatigue Moni- toring", in The 7th International IEEE Conference on Intelligent Trans- portation Systems, pp. 657-662, Oct. 2004

[11] A. B. Albu, B. Widsten, T. Wang, J. Lan and J. Mah, "A Computer Vision-Based System for Real-Time Detection of Sleep Onset in Fatigued Drivers", in 2008 IEEE Intelligent Vehicles Symposium, pp. 25-30, June 2008.

[12] J. Lee, J. Li, L. Liu and C. Chen, "A Novel Driving Pattern Recognition and Status Monitoring System", in First pacific rim symposium, PSIVT 2006, pp. 504-512, December 2006.

[13] A. V. Desai and M. A. Haque, "Vigilance Monitoring for Operator Safety: A Simulation Study on Highway Driving", in Journal of Safety Research, Vol. 37, No. 2, pp. 139-147, 2006.

[14] J. Krajewski, D. Sommer, U. Trutschel, D. Edwards and M. Golz, "Steering Wheel Behavior Based Estimation of Fatigue", in Proceedings of the Fifth International Driving Symposium on Human Factors in Driver Assessment, Training and Vehicle Design, pp. 118-124.

[15] "Saab AlcoKey Helps Drivers", http://www.saabnet.com/tsn/press/061013a.html

[16] A. Heitmann, R. Cuttkuhn, A. Aguirre, U. Trutschel and M. MooreEde, "Technologies for The Monitoring and Prevention of Driver Fatigue", in Proceedings of the Fifth International Driving Symposium on Human Factors in Driver Assessment, Training and Vehicle Design, pp. 81-86.

[17] V. D. Lecce and M. Calabrese, "Experimental System to Support Real- Time Driving Pattern Recognition”, in Advanced Intelligent Computing Theories and Applications With Aspects of Artificial Intelligence Annals of Emergency Medicine, pp. 1192-1199, 2008.

[18] Wikipedia, "GPS" entry, http://en.wikipedia.org/wiki/Global Positioning System.

[19] M. Wilczkowiak, E. Boyer, and P. Sturm, "Camera Calibration and 3D Reconstruction from Single Images Using Parallelepipeds", in Proceed- ings of ICCV, pp. 142-148, 2001. 